\title{
As-required versus regular nebulized salbutamol for the treatment of acute severe asthma
}

\author{
P. Bradding, I. Rushby, J. Scullion, M.D.L. Morgan
}

As-required versus regular nebulized salbutamol for the treatment of acute severe asthma. P. Bradding, I. Rushby, J. Scullion, M.D.L. Morgan. C ERS Journals Ltd 1999.

ABSTRACT: Current British guidelines for the administration of $\beta_{\mathbf{2}}$-agonists in acute severe asthma recommend regular nebulized therapy in hospitalized patients, followed by as-required (p.r.n.) use via hand-held devices after discharge. Since $\beta_{2}$-agonists do not possess anti-inflammatory activity in vivo, and are thus unlikely to influence the rate of recovery from an asthma exacerbation, it was hypothesized that patients given the short-acting $\beta_{2}$-agonist salbutamol on an as-required basis after admission to hospital would recover as quickly as those on regular treatment, but with potential reductions in the total dose delivered.

Forty-six patients with acute severe asthma were randomly assigned to either regular prescriptions of nebulized salbutamol or to usage on a p.r.n. basis, from $24 \mathrm{~h}$ after hospital admission. The primary outcome measures were length of hospital stay, time to recovery, and frequency of salbutamol nebulization from $24 \mathrm{~h}$ after admission to discharge. Secondary outcome measures were treatment side-effects (tremor, palpitations), and patient satisfaction.

Length of hospital stay was reduced in those patients allocated to p.r.n. salbutamol (geometric mean (GM) 3.7 days) versus regular salbutamol (GM 4.7 days). Time taken for peak expiratory flow to reach $75 \%$ of recent best was the same in both groups. There was a highly significant reduction in the number of times nebulized therapy was delivered to the p.r.n. group (GM 7.0, range 1-30) compared with the regular treatment group (GM 14.0, range $4-57 ; p=0.003 ; 95 \%$ confidence interval for ratio of GMs 1.29-3.09). In addition, patients reported less tremor $(p=0.062)$ and fewer palpitations $(\mathrm{p}=0.049)$ in the p.r.n. group. Of the patients in the p.r.n. group who had received regular nebulized therapy on previous admissions $(n=12)$, all preferred the p.r.n. regimen.

Prescribing $\beta_{2}$-agonists on a p.r.n. basis from $24 \mathrm{~h}$ after hospital admission is associated with reduced amount of drug delivered, incidence of side-effects, and possibly length of hospital stay. This has implications for the efficient use of healthcare resources.

Eur Respir J 1999; 13: 290-294.

Acute asthma remains a frequent cause of morbidity throughout the world, and sets a great financial burden on overstretched healthcare resources. In spite of extensive laboratory and clinical research, there is still no firm evidence to indicate the optimal mode of treatment for patients with acute exacerbations of their disease.

Current British guidelines [1] on the treatment of acute severe asthma propose an initial $5 \mathrm{mg}$ dose of salbutamol or $10 \mathrm{mg}$ of terbutaline delivered by nebulizer, followed by regular nebulized doses at four-hourly intervals for patients who are responding. However, one-tenth of this dose delivered by metered-dose inhaler and large volume spacer has been shown to be just as effective $[2,3]$. The British Thoracic Society guidelines on the use of nebulizers in acute severe asthma reflect current UK practice, stating that administration of regular nebulized $\beta_{2}$-agonists should be continued until patients meet the criteria for discharge [4], at which point they are converted to hand-held inhaled therapy. If stable for a further $24 \mathrm{~h}$, patients can then go home.
Dept of Respiratory Medicine and Thoracic Surgery, The Glenfield Hospital, Leicester, UK.

Correspondence: P. Bradding

Dept of Respiratory Medicine and Thoracic Surgery

Glenfield Hospital

Groby Road

Leicester LE3 9QP

UK

Fax: 441162367768

Keywords: Acute asthma

nebulizers

salbutamol

Received: March 91998

Accepted after revision September 271998
In addition to the costs of giving regular nebulized treatment, there are potentially serious adverse effects of high-dose treatment, including the development of hypokalaemia, arterial hypoxaemia, and, in older patients, cardiac ischaemia, all of which may predispose to cardiac arrhythmias and sudden death [5-9]. Furthermore, there is evidence which suggests that longer-term, regular treatment with short-acting $\beta_{2}$-agonists leads to a deterioration in asthma control and may predispose to asthma death $[10-12]$, and it is with this in mind that current guidelines suggest only as-required (p.r.n.) use of these drugs for maintenance medication.

It is likely that the underlying pathology in many patients requiring hospitalization for acute severe asthma is an exacerbation of the airway inflammatory response [1316], and corticosteroids, which have potent anti-inflammatory activity, appear to hasten recovery, with a parallel improvement in inflammatory indices [15-19]. Since $\beta_{2^{-}}$ agonists are apparently devoid of important anti-inflammatory effects in vivo [20], it was hypothesized that patients 
given the short-acting $\beta_{2}$-agonist salbutamol on an asrequired basis after admission to hospital would recover as quickly as those on regular treatment, but with potential reductions in the total dose delivered. A prospective randomized study was therefore performed to evaluate the efficacy of regular versus p.r.n. nebulized salbutamol in the management of patients with acute severe asthma.

\section{Methods}

\section{Study design}

This was an open, prospective, randomized, controlled study. From the length of stay data in the authors' 1994 asthma audit of 50 patients admitted with acute asthma (geometric mean length of stay 4.7 days, range 1-12 days), the number of times nebulized therapy would have been administered when prescribed on a regular basis from $24 \mathrm{~h}$ after admission to $24 \mathrm{~h}$ before discharge was estimated. From this, a sample size of 39 patients was estimated to give the study at least $80 \%$ power at $5 \%$ significance to detect a $33 \%$ difference in the frequency of nebulization between the two intervention groups. Fortysix patients admitted consecutively to the respiratory unit at Glenfield General Hospital with a diagnosis of acute severe asthma were subsequently invited to take part. Fortyfive patients had pre-existing asthma and one patient was a new presentation. Patients were eligible if they were between 17 and 65 yrs of age, and were not using regular nebulized $\beta_{2}$-agonists at home. All subjects included in the study gave their written informed consent and the study was approved by the Leicester Health Authority Ethics Committee, with the proviso that all patients included in the study would receive regular four-hourly nebulizers for the first $24 \mathrm{~h}$, in line with current British guidelines.

On admission, the following variables indicative of asthma severity were recorded before treatment in all patients: pulse, respiratory frequency, transcutaneous oxygen saturation while receiving $40 \% \mathrm{O}_{2}$ via facemask, and peak expiratory flow (PEF) (Wright mini-peak flow meter; Clement Clarke International, London, UK). Measurement of blood gases was optional at the discretion of the admitting physician. Initial treatment was administered in accordance with current British guidelines [1], with $5 \mathrm{mg}$ nebulized salbutamol initially, repeated as required, and continued at four-hourly intervals for $24 \mathrm{~h}$. Consecutive consenting patients were randomized by hospital number so that, from $24 \mathrm{~h}$ after admission, they would receive either regular four-hourly nebulized salbutamol $5 \mathrm{mg}$ (odd numbers) $(\mathrm{n}=24)$, or $2.5-5.0 \mathrm{mg}$ nebulized salbutamol as required (even numbers) $(\mathrm{n}=22)$. Patients in the p.r.n. group were simply instructed to ask for nebulized treatment if they felt it was required, just as they would if they were symptomatic when using regular treatment. Patients in the regular treatment group were also allowed additional nebulized salbutamol if needed. Administration of other medications was left to the discretion of the prescribing physicians who followed the current British guidelines. Thus, all patients received prednisolone $40 \mathrm{mg}$ daily, and received nebulized ipratropium and/or intravenous aminophylline if indicated. When PEF reached $75 \%$ of best and diurnal PEF variation was $<25 \%$, nebulized therapy was discontinued, and normal hand-held therapy prescribed as per British asthma guidelines. The prescribing physicians were unaware of the randomization method which was controlled by one of the investigators (I. Rushby).

The primary outcome measures to be examined were length of stay in hospital, time taken for the PEF to reach $75 \%$ of the best value in the preceding year, and frequency of salbutamol nebulization from $24 \mathrm{~h}$ after admission to discharge. Secondary outcome measures were treatment side-effects (tremor, palpitations).

\section{Data analysis}

Baseline demographic data are expressed as means \pm SEM, or medians and ranges where appropriate. Data for length of stay in hospital, time taken for the PEF to reach $75 \%$ of previous best, number of nebulizations administered, and total salbutamol use from $24 \mathrm{~h}$ after admission were log transformed, and differences between groups explored using the unpaired Student's t-test. These data are presented as geometric means (ranges). Frequency of sideeffects, recorded as present or absent, were compared using Fisher's exact test (two-tailed). The level of statistical significance was accepted as $\mathrm{p}<0.05$.

\section{Results}

All of the 46 patients invited to take part in the study accepted, and no patient withdrew for any reason. Randomization by hospital number resulted in 24 patients receiving regular nebulized salbutamol, and 22 p.r.n. salbutamol. The baseline demographic data of the 46 patients are shown in table 1. Patients randomized to the two groups were well matched at baseline for age, sex, PEF ( $\%$ of best value in last year and $\%$ predicted), pulse, respiratory frequency, requirement for blood gas analysis, length of asthma history, and pre-admission medication. Additional treatment requirements for the first $24 \mathrm{~h}$, and PEF values after $24 \mathrm{~h}$ were also well matched (table 2), indicating that neither group was at a disadvantage at the time randomized treatment was commenced. Following the commencement of randomized treatment $24 \mathrm{~h}$ after admission, 6 $(25 \%)$ patients in the regular group received additional nebulized ipratropium bromide compared with 3 (14\%) patients in the p.r.n. group. A further patient in the p.r.n. group received intravenous aminophylline from 24 to 48 $\mathrm{h}$ after admission.

The outcome data are summarized in table 3. There was a significant reduction in length of hospital stay in patients receiving nebulized salbutamol on a p.r.n. basis (geometric mean 3.7 days, range 2-7 days) compared with those receiving regular medication (geometric mean 4.7 days, range $2-12$ days, $\mathrm{p}=0.044 ; 95 \%$ confidence interval (CI) for ratio of geometric means 1.01-1.62). The time taken for the PEF to reach $75 \%$ of the best value in the last year was similar in the two groups (p.r.n. group geometric mean 2.7 days, range 1-7 days; regular group geometric mean 2.7 days, range $1-8$ days; $p=0.95$ ). There was a highly significant reduction in the number of times nebulized therapy was delivered to the p.r.n. group (geometric mean 7.0, range 1-30) compared with the regular treatment group (geometric mean 14.0, range 4-57; $\mathrm{p}=$ 0.003 ; $95 \%$ CI for the ratio of geometric means 1.29 3.09). Not surprisingly, this was paralleled by a highly 
Table 1. - Demographic data and markers of asthma severity at time of admission while breathing $40 \% \mathrm{O}_{2}$ expressed as mean \pm SEM

\begin{tabular}{lcc}
\hline & $\begin{array}{c}\text { Regular salbutamol } \\
\text { group }(\mathrm{n}=24)\end{array}$ & $\begin{array}{c}\text { p.r.n. salbutamol } \\
\text { group }(\mathrm{n}=22)\end{array}$ \\
\hline Sex F/M & $17 / 7$ & $15 / 7$ \\
Age yrs & $32 \pm 2$ & $28 \pm 2$ \\
(range) yrs & $(18-54)$ & $(17-57)$ \\
Asthma history yrs* & $12.5(0-31)$ & $17.5(1-36)$ \\
Initial PEF \% best & $50.3 \pm 2.7$ & $56.9 \pm 3.4$ \\
Initial PEF \% pred & $45.6 \pm 3.5$ & $47.4 \pm 3.6$ \\
Pulse rate beats·min & $106.5 \pm 3.6$ & $102.5 \pm 3.5$ \\
Respiratory frequency breaths·min ${ }^{-1}$ & $24.9 \pm 1.9$ & $25.1 \pm 1.3$ \\
Oxygen saturation \% & $95.3 \pm 0.5$ & $94.9 \pm 0.5$ \\
Blood gases sampled $n$ & 20 & 14 \\
PCO in those sampled kPa & $4.4 \pm 0.2$ & $4.2 \pm 0.2$ \\
Pre-admission medication $n$ & & \\
Inhaled $\beta_{2}$-agonists & 23 & 22 \\
Inhaled steroids & 18 & 21 \\
Oral steroids & 3 & 3 \\
Theophylline & 1 & 1 \\
\hline
\end{tabular}

*: expressed as median (range). M: male; F: female; PEF: peak expiratory flow; $\mathrm{PCO}_{2}$ : carbon dioxide tension.

significant difference in the total dose of salbutamol delivered by nebulizer to the p.r.n. group (geometric mean $29.4 \mathrm{mg}$, range $5.0-85.0 \mathrm{mg}$ ) compared with the regular group (geometric mean $62.8 \mathrm{mg}$, range 15.0$207.5 \mathrm{mg}$; $\mathrm{p}=0.001 ; 95 \% \mathrm{CI}$ for ratio of geometric means $1.37-3.33)$. PEF recordings on discharge ( $\%$ best), were the same in the two study groups (p.r.n. group mean 92.5 (3.6\%), regular treatment group mean $89.0(2.8 \%), \mathrm{p}=$ $0.45)$, demonstrating that the degree of recovery in the two groups was the same. Tremor and palpitations were reported less frequently in the patients receiving p.r.n. treatment $(18.2 \%$ and $4.5 \%$, respectively) than in those receiving regular treatment $(45.8 \%$ and $29.2 \%$, respectively) ( $\mathrm{p}=0.062$ for tremor, $\mathrm{p}=0.049$ for palpitations). All patients in the p.r.n group who had previously been admitted $(n=12)$ and received regular nebulized treatment in the past said they preferred receiving this therapy on an as-required basis.

Analysis restricted to the more severe patients admitted with PEF $<50 \%$ pred $(n=17$ and $n=15$ in the regular and p.r.n groups, respectively) showed that they did not differ from the study population as a whole in terms of any of the outcome measures.

\section{Discussion}

This study has demonstrated that patients admitted to hospital with acute severe asthma can be treated safely with nebulized salbutamol on an as-required basis, from 24

Table 2. - Additional treatment requirements during the first $24 \mathrm{~h}$ and peak expiratory flow (PEF) values at the end of this period immediately prior to randomization expressed as mean \pm SEM

\begin{tabular}{lcc}
\hline & $\begin{array}{c}\text { Regular } \\
\text { salbutamol } \\
\text { group }(\mathrm{n}=24)\end{array}$ & $\begin{array}{c}\text { p.r.n. } \\
\text { salbutamol } \\
\text { group }(\mathrm{n}=22)\end{array}$ \\
\hline Ipratropium bromide $\mathrm{n}$ & 7 & 4 \\
Intravenous aminophylline $\mathrm{n}$ & 0 & 1 \\
PEF at 24 h \% best & $66.7 \pm 4.0$ & $67.4 \pm 3.0$ \\
\hline
\end{tabular}

$\mathrm{h}$ after admission onwards. In fact, there are several advantages for both the patient and the hospital with this form of management, with potential reductions evident in length of inpatient admission, quantity of nebulized $\beta_{2}$ agonists delivered, and incidence of reported side-effects. Furthermore, patients receiving p.r.n. treatment, who had received regular treatment on previous admissions, preferred the as-required protocol.

Following recent concerns about the safety of $\beta_{2}$-agonists, current advice is that they are reserved for treatment of symptoms on an as-required basis in the community [1]. Exacerbations of asthma requiring hospital admission are likely, in most cases, to be a consequence of potentiation of inflammatory mechanisms within the bronchial mucosa, often in association with viral infection [13-16, 21]. Recovery coincides with resolution of the inflammatory changes $[15,16]$, which is probably the mechanism through which corticosteroids exert their antiasthma effect. As $\beta_{2}$-agonists do not appear to exert important anti-inflammatory effects in vivo [20], it also seems logical that, during asthma exacerbations, they still be used for the treatment of symptoms on an as-required basis, particularly as drug requirements will vary from patient-to-patient. The results of this study clearly support this idea, and demonstrate that the regular administration of nebulized $\beta_{2}$-agonists is unnecessary.

A surprising finding was that length of hospital stay was reduced in the p.r.n. group. The main purpose of monitoring this was to ensure that the p.r.n. protocol was not harmful to patients, which from the data the authors can be confident is not. This difference in hospital stay between the two groups is probably not an adverse effect of prescribing regular nebulized salbutamol, since the time taken for PEF to reach $75 \%$ of the recent best value was almost identical in both groups. This latter observation also indicates that p.r.n treatment was not harmful. Time of discharge from hospital, however, may be influenced by a number of factors, so in this relatively small population it is not possible to be sure that the difference observed between the two groups is related to the treatment interventions. However, the longer stay in the regular 
Table 3. - Outcome variables expressed as geometric mean (range) or mean \pm SEM

\begin{tabular}{lccc}
\hline & $\begin{array}{c}\text { Regular salbutamol } \\
\text { group (n=24) }\end{array}$ & $\begin{array}{c}\text { p.r.n. salbutamol } \\
\text { group (n=22) }\end{array}$ & p-value \\
\hline Length of stay days & 4.7 & 3.7 & 0.044 \\
& $(2-12)$ & $(2-7)$ & 0.95 \\
Time for PEF to reach & 2.7 & $(1-7)$ & 0.001 \\
$75 \%$ of best days & $(1-8)$ & 29.4 & 0.003 \\
Salbutamol delivered mg & 62.8 & $(5.0-85.0)$ & 7.0 \\
Number of nebulizations & $(15.0-207.5)$ & $(1-30)$ & 0.063 \\
Number of patients with: & 14.0 & 4 & 0.049 \\
$\quad$ Tremor & $(4-57)$ & 1 & 0.45 \\
Palpitations & 11 & $92.5 \pm 3.6$ & \\
PEF on discharge \% best & 7 & & 0.0 \\
\hline
\end{tabular}

PEF: peak expiratory flow.

salbutamol group may reflect in part the extra day needed to switch patients to the hand-held inhaled therapy they will take at home, in accordance with current British guidelines $[1,4]$. It would be inappropriate to make financial calculations on the basis of a borderline significant reduction in length of hospital stay. However, the data on drug usage do suggest that the reduced frequency of nebulization will lead to efficiency savings in terms of nursing time, and small but not insignificant financial savings in terms of expenditure on salbutamol. In addition, patients avoid unnecessary treatment, with the result that they experience fewer side-effects and potentially less risk of serious adverse effects.

One potential criticism of this study is that it was not performed blind. This would have required administering a regular nebulized placebo to the as-required treatment group in the form of $0.9 \%$ saline. This, however, may not be without some therapeutic benefit through its potential to hydrate and loosen sticky secretions, which contribute significantly to the airflow obstruction of acute asthma [13], and would not be used subsequently if clinical practice were to be altered. Furthermore, it is likely that most patients could detect the difference between nebulized placebo and salbutamol by the clinical response. What is important is whether performing an open study will have biased the results in favour of the p.r.n. group. The authors believe this is unlikely as, firstly, the attending clinicians were unaware of the randomization method, and so could not have influenced treatment during the first $24 \mathrm{~h}$ of admission. This is supported by the similar needs for additional treatments and the degree of recovery in the two groups based on PEF data after $24 \mathrm{~h}$, and that, after randomization, additional treatment requirements remained the same in the two groups. Furthermore, the attending clinicians had nothing to gain from the outcome of this study and would therefore be unlikely to favour one arm in preference to the other. Secondly, several patients in each study group had been admitted previously, and, as far as they were concerned, regular nebulized $\beta_{2}$-agonists were the standard form of treatment. From the patients perspective it is therefore perhaps more likely that receiving treatment on a p.r.n. basis would be perceived as having the potential to do more harm, and, if anything, might have biased the results in favour of the regular treatment group.

There were four patients in this study who required additional treatment (three nebulized ipratropium, one intra- venous aminophylline) while receiving p.r.n salbutamol. There could be concerns that while the p.r.n. treatment protocol is safe for most patients, these patients at the more severe end of the spectrum could be undertreated and put at risk. However, prescribing treatment at regular fixed intervals for patients with more severe asthma would not necessarily be any safer, since some patients may need nebulized $\beta_{2}$-agonists more frequently than prescribed, or even continuously, and are in effect still receiving them p.r.n. What is important is that the more severe patients receive an appropriate level of medical attention.

In summary, it was demonstrated that nebulized salbutamol administered on an as-required basis from $24 \mathrm{~h}$ after hospital admission for acute severe asthma has advantages over regular nebulized treatment with respect to frequency and dose of drug administered, side-effect profile, patient preference, and possibly length of hospital stay. The authors have altered the prescribing policy in their hospital accordingly, and have not had any adverse events over the 9 months that this has been in practice.

Acknowledgements. The authors would like to thank J.M. Wales, J.B. Cookson, A.J. Wardlaw and I.D. Pavord for allowing us to include their patients in the study, and are grateful to N. Taub, Dept. of Epidemiology and Public Health, University of Leicester, for statistical advice.

\section{References}

1. British Thoracic Society. The British guidelines on asthma management: 1995 review and position statement. Thorax 1997; 52: Suppl. 1.

2. Idris AH, McDermott MF, Raucci JC, Morrabel A, McGorray S, Hendeles L. Emergency department treatment of severe asthma. Metered dose inhaler plus holding chamber is equivalent in effectiveness to nebuliser. Chest 1993; 103: 665-672.

3. Colacone A, Afilalo M, Wolkove N, Kreisman H. A comparison of albuterol administered by metered dose inhaler (and holding chamber) or wet nebuliser in acute asthma. Chest 1993; 104: 835-841.

4. British Thoracic Soceity. The nebuliser project group of the British Thoracic Society standards of care committee. Current best practice for nebuliser treatment. Thorax 1997; 52: Suppl. 2. 
5. Palmer KNV, Diament ML. Effects of salbutamol on spirometry and blood gas tensions in bronchial asthma. $B M J$ 1969; 1: 31-32.

6. Smith SR, Ryder C, Kendall MJ, Holder R. Cardiovascular and biochemical responses to nebulised salbutamol in normal subjects. Br J Clin Pharmacol 1984; 18: 641-644.

7. Neville E, Corris PA, Vivian J, Nariman S, Gibson GJ. Nebulised salbutamol and angina. BMJ 1982; 285: 796797.

8. Higgins RM, Cookson WO, Lane DJ, John SM, McCarthy GL, McCarthy ST. Cardiac arrhythmias caused by nebulised beta-agonist therapy. Lancet 1987; ii: 863-864.

9. Robin ED, McCauley R. Sudden cardiac death in bronchial asthma, and inhaled beta-adrenergic agonists. Chest 1992; 101: 1699-1702.

10. Crane J, Pearce N, Flatt A, et al. Prescribed fenoterol and death from asthma in New Zealand. Lancet 1989; i: 917922.

11. Spitzer WO, Suissa S, Ernst P, et al. The use of $\beta$-agonists and the risk of death and near-death from asthma. $N$ Engl J Med 1992; 326: 501-506.

12. Sears MR, Taylor DR, Print CG, et al. Regular inhaled beta agonist treatment in bronchial asthma. Lancet 1990; 336: 1391-1396.

13. Dunnill MS. The pathology of asthma with special reference to changes in the bronchial mucosa. J Clin Pathol 1960; 13: 27-33.
14. Synek M, Beasley R, Frew AJ, et al. Cellular infiltration of the airways in asthma of varying severity. Am J Respir Crit Care Med 1996; 154: 224-230.

15. Twaddell SH, Gibson PG, Carty K, Woolley KL, Henry RL. Assessment of airway inflammation in children with acute asthma using induced sputum. Eur Respir J 1996; 9: 2104-2108.

16. Pizzichini MM, Pizzichini E, Clelland L, et al. Sputum in severe exacerbations of asthma: kinetics of inflammatory indices after prednisone treatment. Am J Respir Crit Care Med 1997; 155: 1501-1508.

17. Anonymous. Report to the Medical Research Council by the subcommittee on clinical trials in asthma. Controlled trial of the effects of cortisone acetate in status asthmaticus. Lancet 1956; ii: 798-803.

18. Fanta $\mathrm{CH}$, Rossing TH, McFadden ER. Glucocorticoids in acute asthma. A critical controlled trial. $\mathrm{Am} \mathrm{J} \mathrm{Med}$ 1983; 74: 845-851.

19. Haskell RJ, Wong BM, Hansen JE. A double-blind randomised clinical trial of methylprednisolone in status asthmaticus. Arch Intern Med 1983; 143: 1324-1327.

20. Bradding P. Anti-inflammatory effects of long-acting $\beta$ agonists in asthma. Clin Exp Allergy 1996; 26: 648-655.

21. Johnston SL, Pattemore PK, Sanderson G, et al. Community study of role of viral infections in exacerbations of asthma in 9-11 year old children. BMJ 1995; 310: $1225-1228$. 